\title{
mRNA Biomarkers for Detection of Oral Squamous Cell Cancer
}

\author{
Fatemeh Rashid ${ }^{1}$, Tahereh Naji ${ }^{2}$, Abdolreza Mohamadnia ${ }^{3}$, Naghmeh Bahrami ${ }^{4}$
}

${ }^{1}$ Department of Molecular and Cellular Sciences, Faculty of Advanced Sciences and Technology, Pharmaceutical Sciences Branch, Islamic Azad University, Tehran, Iran. ${ }^{2}$ Department of Basic Sciences, Faculty of pharmacy, Pharmaceutical Sciences Branch, Islamic Azad University, Tehran, Iran. ${ }^{3}$ Chronic Respiratory Diseases Research Center, National Research Institute of Tuberculosis and Lung Diseases (NRITLD), Shahid Beheshti University of Medical Sciences, Tehran, Iran; Department of Biotechnology, School of Advanced Technologies in Medicine, Shahid Beheshti University of Medical Sciences, Tehran, Iran. ${ }^{4}$ Craniomaxillofacial Research Center, Tehran University of Medical Sciences, Tehran, Iran; Department of Oral and Maxillofacial Surgery, School of Dentistry, Tehran University of Medical Sciences, Tehran, Iran.

\begin{abstract}
Objectives: Cancer is one of the main causes of death in the world. Changes in the expression of CK19 and CEA genes in peripheral blood of OSCC patients were examined for early diagnosis. Methodology: The participants were 36 patients and 36 normal individuals. CK19 and CEA of blood serum were measured through Real-Time PCR. The relationship of the biomarkers with tumor staging and cancer development was examined. Results: Comparison of the two groups of participants using t-test indicated no significant difference in terms of mean age. CK19 marker was positive in 19 participants of the patient's group $(n=36)$, which meant the sensitivity of the marker was 53\%. In addition, the marker was positive in eight participants of the normal group $(n=36)$. CEA marker was positive in 26 participants of the patient's group $(n=36)$, which meant the sensitivity of the marker was $72 \%$. Moreover, the marker was positive in 11 participants in the normal group $(n=36)$. Conclusion: In general, the study introduced a screening test for early diagnosis of OSCC. To have evidence with more reliability, future studies should be carried out with larger sample groups.
\end{abstract}

Keywords: Oral squamous cells carcinoma- early diagnosis- CK19- CEA

Asian Pac J Cancer Care, 3 (1), 1-4

\section{Introduction}

Oral squamous cells carcinoma (OSCC) is featured with a high level of topical invasion, metastasis, and high mortality rate. Despite advances in therapeutic techniques like chemotherapy, radiotherapy, and surgery over the past few decades, the mortality rate of the disease still is high $[1,2]$. It is a rare disease with high mortality rate [2].

Etiology of OSCC is multi-factors and it occurs through a multi-stage process. Changes and damages affect and destabilize DNA [3]. OSCC is the most common oral cancer with epithelium causes in the oral cavity. The disease covers $90 \%$ of oral malignancies [3].

Smoking (Cigarette and tobacco) and alcoholic drinks are the main factors effective on the development of oral cancer. Studies have shown that more than $50 \%$ of the patients smoke and the prevalence of the disease in the
Submission Date: 10/12/2017Ａcceptance Date: 12/05/2017

smokers is 2-3 times of the non-smokers [4].

Diagnosing cancer through finding an accurate and effective relationship between biomarkers and the clinical symptoms of the disease and designing a non-invasive diagnosis method at the early stages of the disease is a serious challenge [5]. Diagnostic and prognostic biomarkers help the physicians to identify the cases with the higher risk of cancer in early examinations. Through this, the physician has the opportunity to adopt the most effective medication [6-10]. Recently, biomarkers have drawn a great deal of attention for early diagnosis of cancer $[11,12]$.

Cytokeratin 19 (CK19) is a member of intermediate stand proteins family (Keratin family). The family includes 20 types of polypeptides, plays the main role in preserving the integrity of epithelial cells, and it is one of the highly effective markers in diagnosing and controlling

Corresponding Author:

Dr. Naghmeh Bahrami

Craniomaxillofacial Research Center, Tehran University of Medical Sciences, Tehran, Iran; Department of Oral and Maxillofacial Surgery,

School of Dentistry, Tehran University of Medical Sciences, Tehran, Iran.

Email: n-bahrami@sina.tums.ac.ir 
tumors $[1,13]$.

Carcinoembryonic antigen (CEA) is a glycoprotein of the cell membrane that acts as a tumor marker for diagnosing cancers. It is effective on the adhesiveness of cell and it is usually synthesized during embryo development and stops functioning shortly before child-birth [4]. The increase of serum level of CEA is a prognostic of the disease. Still, using more than one marker and carrying out molecular tests to examine mutations are more effective treatment methods [2].

\section{Materials and Methods}

Totally, 36 patients at Cancer Institute, Tehran University of Medical Sciences diagnosed as suspect OSCC cases and 36 volunteer healthy individuals (after being examined by a specialist) took part in the study.

By patient we refer to an individual at 1, 2, or 3rd stage of the disease -i.e. without distance recurrence metastasis, who has received no medical intervention (e.g. chemotherapy, radiotherapy or surgery). The participants in both groups were identical in terms of age and gender.

Pleural fluid sample $(10 \mathrm{ml})$ was taken from all the participants. The top $2 \mathrm{ml}$ sample was removed from the study due to the risk of contamination with epithelial cells. The rest of the samples were immediately sent to a lab for RNA extraction. The volume of sample was determined based on the sensitivity of RT-PCR technique.

\section{$R N A$ extraction}

The process was carried out using RNeasy Midi Kit (Qiagen Cat no. 75144). At first, red blood cells (if any) were lysed by lysis buffer. After centrifuge, the cell mass was rinsed with PBS accurately and the total mRNA was extracted through the extraction process.

\section{cDNA development}

The process was carried out using Viva 2-steps RT-PCR Kit (Cat no.RTPL12). The main elements needed for reverse transcription were provided in RT Primer Mix framework and the reserve transcription was done in a kit.

Quality and quantity of RNA and cDNA were checked at the end of each stage using Nanodrop Spectrophotometer (Bio-Tek, USA).

\section{Designing special primer in AllelleID6}

The special primer for each marker was designed in AlleleID6 and ordered to the producer. Table 1 lists the parameters and required volumes for the final Real-time reaction.

\section{Real-time RT-PCR}

HotTaq EvaGreen qPCR Mix (Cat No.BT11101- Sina

Colon Co.) was used. The elements of RT-PCR were:

a. Pattern sequence $(2 \mu \mathrm{l})$;

b. Master mix $(4 \mu 1)$;

c. The primer prepared based on the optimum concentration determined in set up tests; and d. Distilled deionized water to increase the solution volume to $20 \mu 1$.

Temperature and time period of the reaction were determined based on the instruction on the kit. The results of each reaction were interpreted based on Amplification and melt curves.

\section{Data analyses}

Means scores of the two groups were compared using t-test and the percentage of positive markers in the two groups of the participants was compared using the Two-sample binomial test in SPSS $(20)(\mathrm{p} \leq 0.05)$.

\section{Results}

\section{Findings}

The participants included the patients $(\mathrm{n}=36)$ and normal groups $(n=36)$. The results of t-test showed no significant differences in terms of mean age -i.e. age was not a confounding factor Table 3 .

\section{RT-PCR results}

CEA marker in the patient's group was positive in 26 cases, which means $72 \%$ sensitivity. A number of the positive cases in the normal group was 11 out of 36 . Comparison of the positive cases in the two groups using two-sample binomial test indicated a significant difference between the two groups $(\mathrm{P}$-value $=0.025)$.

CK19 markers in the patient's group were positive in 19 patients, which means 53\% sensitivity. A number of positive cases in the normal group was eight out of 36 . Comparison of the positive cases in the two groups using two-sample binomial test indicated a significant difference between the two groups $(\mathrm{P}-\mathrm{value}=0.011)$ Figure 1 .

\section{Difference between the markers in the two groups}

The relative level of difference between the markers in the two groups of patients and normal individuals was measured for CEA-mRNA and CK19-mRNA through $\Delta \Delta c t$ method. Two to the power of $\Delta \Delta$ ct yields the difference between the markers. As the results showed, the number of primary copies of CEA marker in the patient's group was 2.14 times of the normal group. In addition,

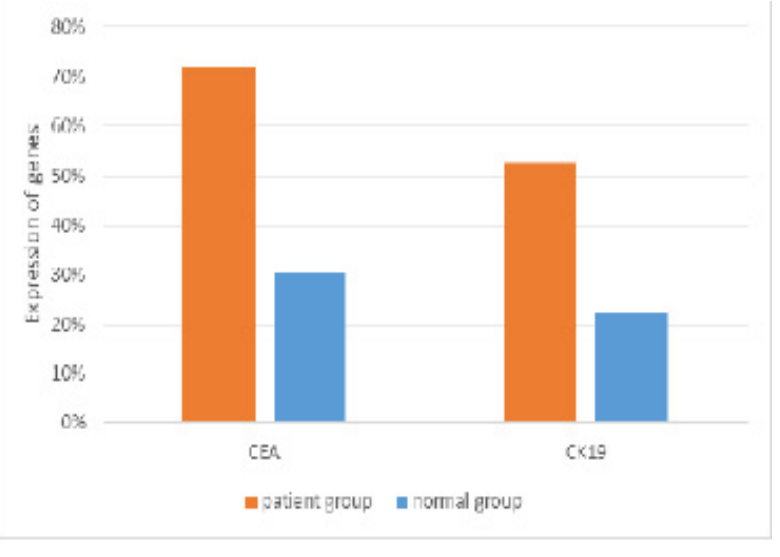

Figure 1. Evaluation of CEA and CK19 mRNA Expression in the Patients Group and Normal Group 
Table 1. Specification of the Primers Using in Real-time RT-PCR

\begin{tabular}{lccc}
\hline Parameter & CEA-mRNA & CK19-mRNA & 18s rRNA \\
\hline F primer & ACCCTGGATGTCCTCTATGG & TCCGAACCAAGTTTGAGAC & GTAACCCGTTGAACCCCATT \\
Length of primer & 20 & 19 & 20 \\
R primer & CAGGCATAGGTCCCGTTATTA & AATCCACCTCCACACTGA & CCATCCAATCGGTAGTAGCG \\
Length of primer & 21 & 18 & 20 \\
Length of proliferation piece & 174 & 222 & 152 \\
Optimum annealing temperature & $61.2^{\circ} \mathrm{C}$ & $58.4^{\circ} \mathrm{C}$ & $53.5^{\circ} \mathrm{C}$ \\
\hline
\end{tabular}

the number of primary copies of the CK19 marker in the patient's group was 1.52 times of the normal group Figure2.

\section{Discussion}

OSCC is the eighth prevalent cancer in men and fifteenth prevalent cancer in women [14]. It encompasses tumors of lips, tongue, gum, palate, saliva glands, tonsils, oropharyngeal, nasopharynx, and hypopharynx. More than $90 \%$ of oral cancers are OSCC and about $9 \%$ are saliva glands, sarcoma, and lymphoma carcinoma [14].

Since the five years, survival rate is directly related to the stage of disease at the time of diagnosis, early preventive and intervention treatments not only attenuates the severity of the disease but also stops the progress of the disease. Thereby and given the growing prevalence of oral cancer and that the disease is the cause of death of many, early diagnosis of the disease is imperative [15].

Biomarkers have drawn a great deal of attention for early diagnosis of cancer recently [11]. In fact, the biomarkers are key tools to study, follow up, and diagnose cancer [16].

In addition, tumor markers provide a way to supervise effectiveness of therapeutic measures [17]. Detailed knowledge of biomarkers of different disease including cancer is one of the key requirements in medicine. One of the main usages of the biomarkers is to determine the stage of cancer [18]. Methods for early diagnosis of OSCC were examined in this paper.

CK19 is a member of the large family of intermediate strand protein (Keratin family). There are more than 20 polypeptides in the family and their main role is to preserve the integrity of epithelial cells. They are one of the key markers in diagnosing and controlling tumors [1].

CEA is a glycoprotein of the cell membrane and a tumor marker for diagnosing cancers. It plays a role in adhesiveness of cells; it is normally synthesized during embryo development and stops functioning shortly before childbirth [2]. Increase in serum level of CEA

Table 2. RT-PCR Temperature and Time Period

\begin{tabular}{lcc}
\hline Real-time step & Temperature & Duration \\
\hline Initial activation & $95^{\circ} \mathrm{C}$ & $5 \mathrm{~min}$ \\
& $40 \mathrm{cycles}$ & \\
Denaturation & $59^{\circ} \mathrm{C}$ & $15 \mathrm{~s}$ \\
Annealing & $56-60^{\circ} \mathrm{C}$ & $60 \mathrm{~s}$ \\
Extension & $72^{\circ} \mathrm{C}$ & $20 \mathrm{~s}$ \\
\hline
\end{tabular}

is a prognosis of the disease progress. Still, surveying more than one tumor marker simultaneously and carrying out molecular tests to spot mutation is a more reliable way to diagnose and treat cancers [1].

In a study on 60 cases with OSCC and 20 normal cases, the VEGF-A levels of serum and tissue were determined through ELISA and immunohistochemistry methods respectively using antibodies against VEGF-A. The expression of VEGF-A mRNA was analyzed through PCR method and the results indicated 50 times increase in the expression [19]. CEA-mRNA and CK19-mRNA of the biomarkers were surveyed and the results showed an increase of the biomarkers in OSCC cases.

Surveys by Zhu G et al. (2004) of the expression of CK19-mRNA and CEA-mRNA in the peripheral blood of NSLC patients showed a positive expression rate of $50 \%$ for CK19-mRNA, $40 \%$ for CEA-mRNA, and $43 \%$ for the both. They concluded that CK19-mRNA and CEF-mRNA were proper biomarkers to diagnose micro-metastasis. Similar results were obtained by a recent study on OSCC [20].

Guo Y et al. (2009) surveyed the expression of CEA mRNA, CK20, and CK19 of peripheral blood in lung carcinoma patients through nested RT-PCR. They reported the positive expression of $41 \%$ of CK20,36.1\% of CK19 and $48.2 \%$ of CEA mRNA; in addition, $73.5 \%$ of the samples had at least one positive marker that was related to metastasis $(\mathrm{P}<0.05)$ [21].

There were $72 \%$ positive CEA mRNA marker and $53 \%$ positive CK 19 mRNA marker in OSCC cases. In this regard, there was a significant difference between the patients and normal groups.

In summary, the results introduced

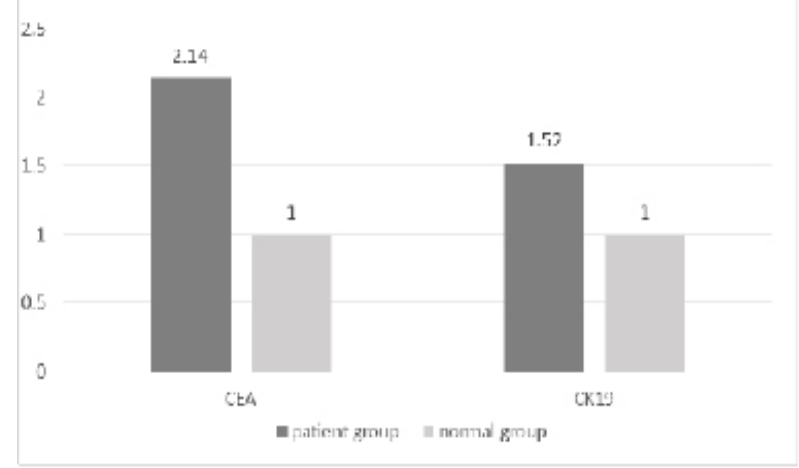

Figure 2. Difference in Expression of CEA, CK19 Genes in the Patients Group and Normal Group 
Table 3. Mean age in the Patients and Healthy Individuals Groups (t-test)

\begin{tabular}{lccc}
\hline & \multicolumn{3}{c}{ Age(years) } \\
Main group & Range & Mean & SD \\
\hline Patients $(\mathrm{n}=40)$ & $24-68$ & 45.65 & 10.33 \\
Healthy $(\mathrm{n}=40)$ & $25-70$ & 49.04 & 11.57 \\
\hline
\end{tabular}

$\mathrm{p}$-value $=0.411 ;(\mathrm{SD}=$ standard deviation $)$

a diagnostic-screening test for early diagnosis of OSCC in the early stages. To have results with higher reliability, further studies with larger sample groups are recommended.

\section{References}

1. Jou Y-J, Lin C-D, Lai C-H, Tang C-H, Huang S-H, Tsai M-H, et al. Salivary zinc finger protein 510 peptides as a novel biomarker for detection of oral squamous cell carcinoma in early stages. Clinica Chimica Acta. 2011;412(15):1357-65.

2. Jou Y-J, Lin C-D, Lai C-H, Chen C-H, Kao J-Y, Chen S-Y, et al. Proteomic identification of salivary transferrin as a biomarker for early detection of oral cancer. Analytica Chimica Acta. 2010;681(1):41-8.

3. Grunnet M, Sorensen J. Carcinoembryonic antigen (CEA) as a tumor marker in lung cancer. Lung Cancer. 2012;76(2):13843.

4. De R. Human cytogenesis: constitutional analysis. London: Oxford; 2001.

5. Benlloch S, Galbis J, Peiro F, Alenda C, Rodriguez-Paniagua J, Sanchez-Paya J, et al. Role of CEA, PLUNC and CK19 mRNA expression in lymph nodes from resected stage I non-small cell lung cancer (NSCLC) patients (p) as markers of occult micrometastasis. A pilot study. Journal of Clinical Oncology. 2005;23(16_suppl): 9654-.

6. Xu D, Li X-f, Zheng S, Jiang W-z. Quantitative real-time RT-PCR detection for CEA, CK20 and CK19 mRNA in peripheral blood of colorectal cancer patients. Journal of Zhejiang University-Science B. 2006;7(6): 445-51.

7. West L, Vidwans SJ, Campbell NP, Shrager J, Simon GR, Bueno R, et al. A novel classification of lung cancer into molecular subtypes. PLoS One. 2012;7(2): e31906.

8. Bahrami N GM, Jamaati HR, Mohamadnia A, Dargahi H, Kazempour dizaji M,Khosravi A, Heshmatnia J, Vahabi P,Bahrami NA. Expression of two essential mRNA biomarker in the peripheral blood as possible biomarkers for diagnosis of non-small cell lung carcinoma. MINERVA PNEUMOLOGICA. 2016 55(3): 31-6.

9. Ghadimi K, Bahrami N, Fathi M, Farzanegan B, Naji T, Emami M, et al. Diagnostic value of LunX mRNA and CEA mRNA expression in pleural fluid of patients with non-small cell lung cancer. Minerva Pneumologica. 2017;56:90-5.

10. Karimi S BN, Sharifi K, Daustany M, Baghbani-Arani F, Kazempour M, et al. Investigating gene expression level of MUC1 and CEA in pleural fluid of NSCLC lung cancer patients with the real-time RT-PCR method. MINERVA PNEUMOLOGICA. 2017; 56(1): 18-24.

11. Honda K, Ono M, Shitashige M, Masuda M, Kamita M, Miura $\mathrm{N}$, et al. Proteomic approaches to the discovery of cancer biomarkers for early detection and personalized medicine. Japanese journal of clinical oncology. 2012;43(2):103-9.

12. Jamaati H, Bahrami N, Tabarsi P, Khosravi A, Kiani A, Abedini A, et al. Multi-Gene Expression in Anthracosis of the Lungs as One of the Risk Factors for Non-Small Cell
Lung Cancer. Asian Pacific journal of cancer prevention: APJCP. 2017;18(11): 3129.

13. Moshref Behzad N BN, Farzanegan B, Fathi M, Zareh Karizi S, Mohamadnia A. Expression of CK19-mRNA and CEA -mRNA biomarkers in pleural fluid of patients with non-small cell lung cancer. MINERVA PNEUMOLOGICA. 2017;56.

14. Kacher J. Oral and maxillofacial pathology. Case of the month. Histoplasmosis. Texas dental journal. 2013;130(3): 198, 232.

15. Almeida MI, Reis RM, Calin GA. MicroRNA history: discovery, recent applications, and next frontiers. Mutation Research/Fundamental and Molecular Mechanisms of Mutagenesis. 2011;717(1): 1-8.

16. Sarandakou A, Protonotariou E, Rizos D. Tumor markers in biological fluids associated with pregnancy. Critical reviews in clinical laboratory sciences. 2007;44(2): 151-78.

17. Duffy MJ, Duggan C, Keane R, Hill AD, McDermott E, Crown J, et al. High preoperative CA 15-3 concentrations predict adverse outcome in node-negative and node-positive breast cancer: a study of 600 patients with histologically confirmed breast cancer. Clinical chemistry. 2004;50(3):55963.

18. Karimi S, Mohamadnia A, Nadji SA, Yadegarazari R, Khosravi A, Bahrami N, et al. Expression of two basic mRNA biomarkers in the peripheral blood of patients with non-small cell lung cancer detected by real-time rt-PCR, individually and simultaneously. Iranian biomedical journal. 2015;19(1): 17.

19. Nayak S, Goel MM, Chandra S, Bhatia V, Mehrotra D, Kumar S, et al. VEGF-A immunohistochemical and mRNA expression in tissues and its serum levels in potentially malignant oral lesions and oral squamous cell carcinomas. Oral oncology. 2012;48(3): 233-9.

20. Zhu G, Liu D, Wang X, Chen J. Detection of CK19 and CEA mRNA expression for the diagnosis of peripheral blood micrometastases in patients with non-small cell lung cancer. Zhongguo Fei ai za zhi $=$ Chinese journal of lung cancer. 2004;7(3): 226-9.

21. Guo Y, Wang J, Huang P. Clinical Significance of CK20, CK19, CEA mRNAs in Peripheral Blood from Lung Cancer Patients. Zhongguo Fei ai za zhi= Chinese journal of lung cancer. 2009;12(9): 1013-7.

\section{(i) (9)}

This work is licensed under a Creative Commons AttributionNon Commercial 4.0 International License. 